\title{
A Novel System for Tracking Pedestrians Using Multiple Single-Row Laser-Range Scanners
}

\author{
Huijing Zhao and Ryosuke Shibasaki, Member, IEEE
}

\begin{abstract}
In this research, we propose a novel system for tracking pedestrians in a wide and open area, such as a shopping mall and exhibition hall, using a number of single-row laser-range scanners (LD-A), which have a profiling rate of $10 \mathrm{~Hz}$ and a scanning angle of $270^{\circ}$. LD-As are set directly on the floor doing horizontal scanning at an elevation of about $20 \mathrm{~cm}$ above the ground, so that horizontal cross sections of the surroundings, containing moving feet of pedestrians as well as still objects, are obtained in a rectangular coordinate system of real dimension. The data of moving feet are extracted through background subtraction by the client computers that control each LD-A, and sent to a server computer, where they are spatially and temporally integrated into a global coordinate system. A simplified pedestrian's walking model based on the typical appearance of moving feet is defined and a tracking method utilizing Kalman filter is developed to track pedestrian's trajectories. The system is evaluated through both real experiment and computer simulation. A real experiment is conducted in an exhibition hall, where three LD-As are used covering an area of about $60 \times 60 \mathrm{~m}^{2}$. Changes in visitors' flow during the whole exhibition day are analyzed, where in the peak hour, about 100 trajectories are extracted simultaneously. On the other hand, a computer simulation is conducted to quantitatively examine system performance with respect to different crowd density.
\end{abstract}

Index Terms-Feet, open area, pedestrian, single-row laser-range scanner, tracking.

\section{INTRODUCTION}

A NALYZING or monitoring human activities, such as counting the number of passengers, or measuring their trajectories, is considered very useful in various fields, such as building security, planning and management assistant in shopping malls, railway stations, and so on. So far, motion analysis with video data has been a major method to collect such data. A good survey for visual-based surveillance can be found in [2]. Following are several examples that target tracking a relatively large crowd in an open area. Regazzoni and Tesei [7] described a video-based system for people counting over time and detecting overcrowded situations in underground railway stations. Schofield et al. [9] developed a lift-aiding system by counting the number of passengers waiting at each floor. Uchida et al. [12] tracked pedestrians on the street. Sacchi et al. [8] proposed a monitoring application, where crowd flow in an outdoor tourist site is counted from video image. Pai et al. [5] proposed a system of detecting and

Manuscript received June 10, 2003; revised April 15, 2004. This paper was recommended by Associate Editor R. Popp.

The authors are with the Center for Spatial Information Science, University of Tokyo, Tokyo 153-8505, Japan (e-mail: chou@skl.iis.u-tokyo.ac.jp).

Digital Object Identifier 10.1109/TSMCA.2005.843396 tracking pedestrians on a crossroad to prevent traffic accidents. In such systems, video cameras are set in restricted positions to reduce occlusions. Image resolutions and viewing angles are quite limited, due to such camera setting, so that the moving object that has less image pixels might be failed in tracking. Still, the always change of illumination and weather condition is another major obstacle to the reliability and robustness of visual-based systems. In order to cover a large area, multiple cameras are used. Whereas, the data from different cameras are difficult to be combined, especially in a real-time process, since it requires accurate calibration and complicated calculation between different perspective coordinate systems. Up to now, applications of visual-based surveillance are subjected to the extraction of rather few objects in limited environments. On the other hand, single-row laser-range scanner is a new sensor technology, which profiles surroundings using eye-safe laser (class 1A, near-infrared spectrum), measures range distances to target objects according to, e.g., time-of-flight at each controlled beam direction. In recent years, single-row laser-range scanner (briefly called "laser scanner") with a high scanning rate, wide viewing angle, and long-range distance has been developed, and can be bought with a rather low price on market. It attracts more and more attentions in the field of moving object detection and tracking. Applications can be found in [11], where a laser scanner is set on a car to monitor a traffic scene; in [6], where a laser scanner is set on a wheel-chair to track surrounding people, aimed at helping handicapped people traveling through a crowded environment, such a railway station during rush-hour; and in [1], where a laser-based people tracking is presented.

In this paper, we propose a novel tracking system aimed at real-time monitoring of pedestrians' behaviors in an environment, such as a railway station, shopping mall, exhibition hall, etc. A number of single-row laser scanners are used to cover a large area, while reducing occlusions. Distributed data from different laser scanners are spatially and temporally integrated on the air. A pedestrian's walking model is defined, and a tracking method utilizing Kalman filter (e.g., [3], [8], and [13]) is developed. Major differences between our system with that of Fod et al. [1] are that we put laser scanners on the ground level, monitoring pedestrians' feet, and track the pattern of their rhythmic swing feet. Whereas, Fod et al. [1] set laser scanners targeting the waist height of average walking people, where occlusion from waist level is much higher than feet level, and range reflections from not only swinging arms, but also other unpredictable factors, e.g., hand bag, coats, etc., are difficult to be modeled for an accurate tracking. The paper is organized as follows. Section II outlines the sensor system, data acquisition, 


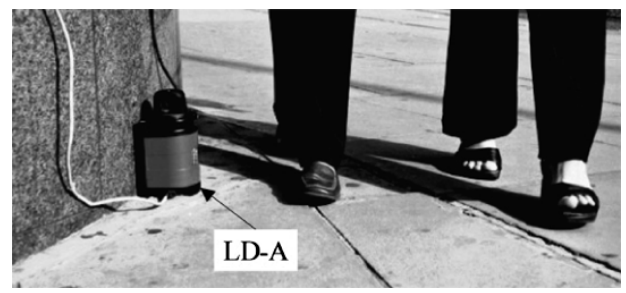

Fig. 1. LD-A at an experimental site.

and distributed data's integration. Section III defines a pedestrian's walking model, followed by an address to the Kalman filter-based tracking algorithm. Section IV examines the system through a whole day experiment at an exhibition hall, and evaluates system performance in different situations through computer simulation.

\section{Outline OF THE System}

\section{A. Single-Row Laser-Range Scanner and Moving Objects Extraction}

Single-row laser-range scanners (LD-A) produced by IBEO Lasertechnik, are exploited (see Fig. 1). In one laser scan, LD-A profiles 1080 range distances equally in $270^{\circ}$ on the scanning plane, which can be easily converted to rectangular coordinates (laser points) in the sensor's local coordinate system. A blind area of $90^{\circ}$ exists due to the hardware configuration. LD-A has a maximum range distance of $70 \mathrm{~m}$ and an average distance error of $3 \mathrm{~cm}$. The frequency of LD-A is $10 \mathrm{~Hz}$, implying that it captures ten laser scans per second. In this paper, LD-As are set doing horizontal scanning at ground level, so that cross sections at the same horizontal level containing the data of moving (e.g., feet) as well as still objects (e.g., building walls, desks, chairs, and so on) are obtained in a rectangular coordinate system of real dimension.

A background image containing still objects only is generated and updated at every time interval (e.g., every $30 \mathrm{~min}$ ) as follows. For each beam direction, a histogram is generated using the range values measured at the direction in all laser scans. A pick value above a certain distinction (percentage) is found out, which tells that an object is continuously measured at the direction with the distance, so that it sounds like a still one. The background image is made up of the pick values at all beam directions. The number of laser scans that are used in background-image generation, and the time interval for background-image updating are decided case by case, according to the environment being measured. In the case that physical layout of the environment does not change often (e.g., exhibition hall and railway station), a background image is generated previously and not updated on the air to avoid mishandling of the range values.

Whenever a new laser scan is recorded, background-image subtraction is conducted at the level of each beam direction. If the difference between two range values is larger than a given threshold (considering the fluctuation of range measurement), the newly measured range value is extracted as the data of a moving object. Fig. 2 shows a sample laser scan, where laser points are colored through background subtraction.

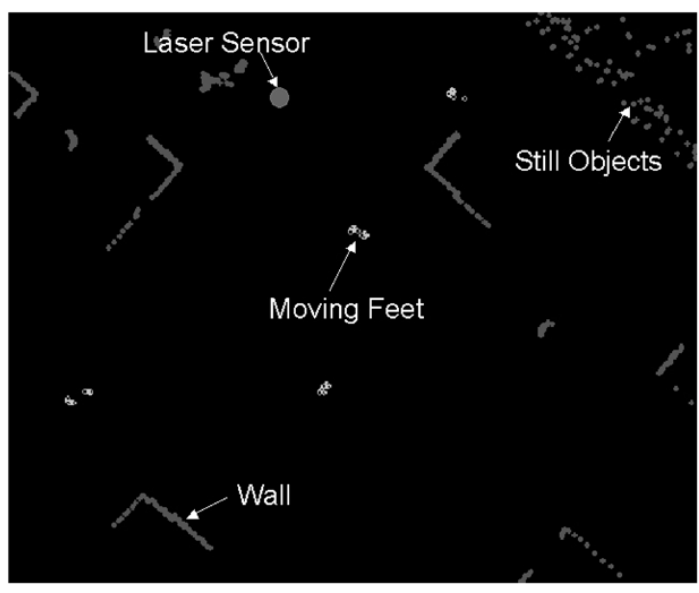

Fig. 2. Sample frame, laser points are colored through background subtraction.

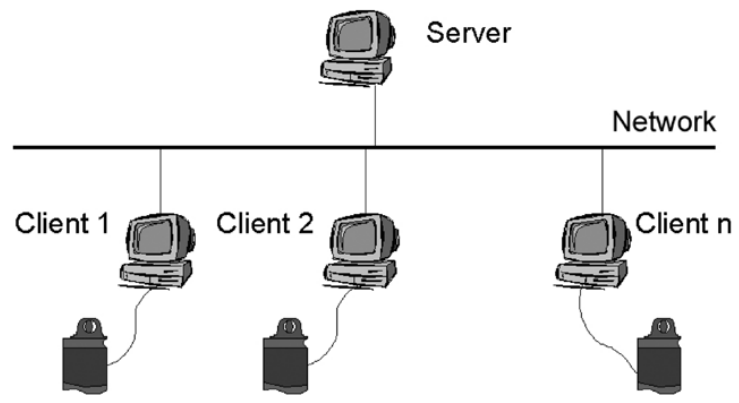

Fig. 3. Integration of multiple laser scanners through network connection.

\section{B. Integration of Multiple Laser Scanners}

A number of laser scanners are exploited, so that a relatively large area can be covered, while occlusions and crossing problem could be solved to some extent. Each laser scanner is located at a separate position and controlled by a client computer. All client computers are connected through a LAN cable to a server computer (see Fig. 3), which gathers the laser points of moving objects from all client computers, and tracks trajectories.

Since laser points from each laser scanner are recorded at a laser scanner's local coordinate system with client computer's local time, they are integrated to a global coordinate system before being processed for tracking, where integration is conducted in spatial ( $x, y$ axes) and temporal (time-axis) levels.

Locations of laser scanners are elaborately planned. All laser scanners form an interconnected network. Laser scans keep a degree of overlay between each others. Relative transformations between the local coordinate system of neighboring laser scanners are calculated by pair-wise matching their background images using the measurements to common objects. In this case, common features in the overlapping area are too few for automated registration, an initial value is first assigned through manual operation, followed by an automated fine-tuning. Assigning an initial value to laser scanners' relative pose is not a tough task here, as two-dimensional (2-D) laser scans are assumed to coincide in the same horizontal plane, operators can shift and rotate one laser scan on the other one to find the best matching between them. Specifying one local coordinate system as the global one, transformations from each local coordinate 


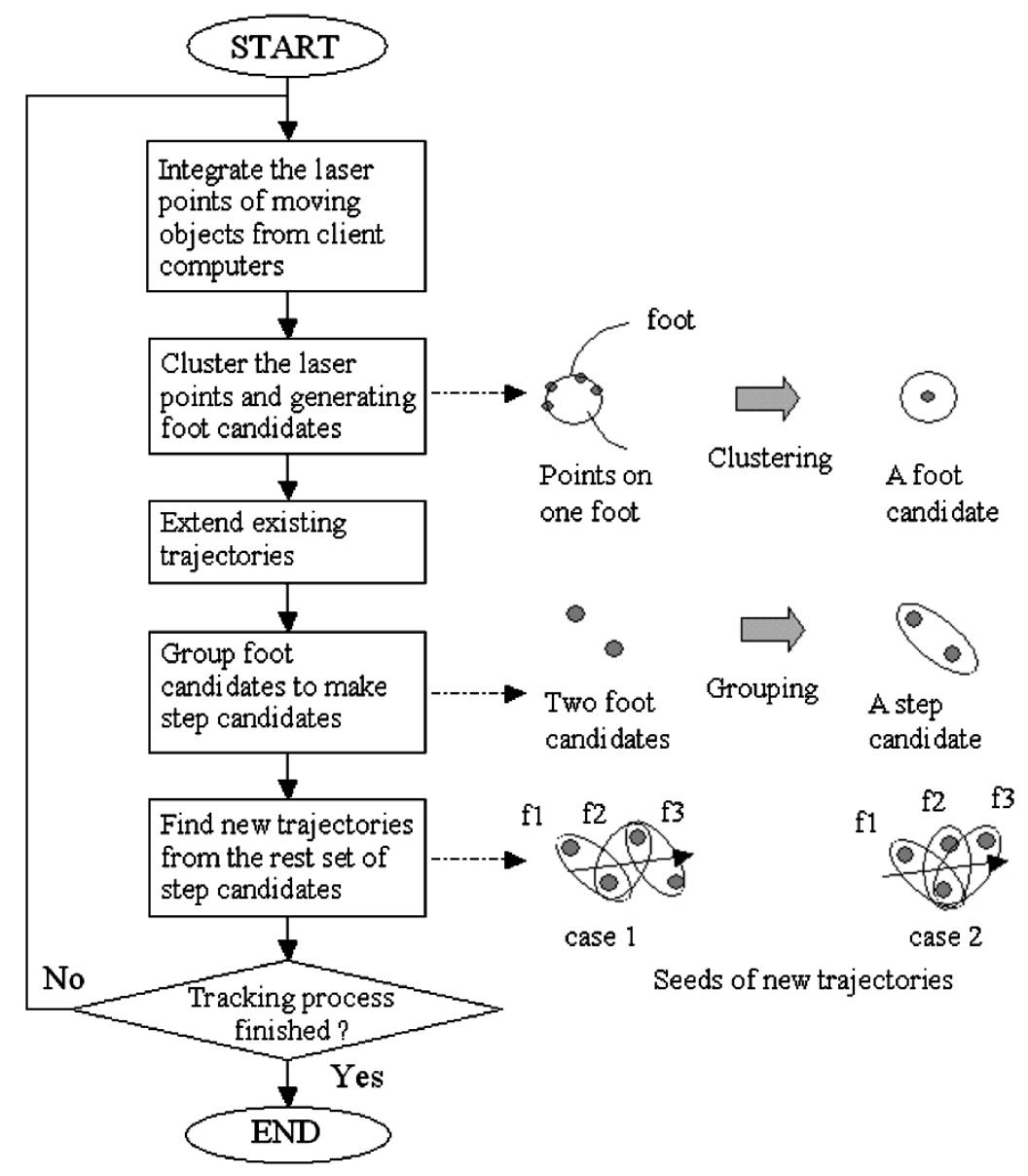

Fig. 4. Flow of the tracking process.

system to the global one are calculated by sequentially aligning the relative transformations, followed by a least-square-based adjustment to solve the error-accumulation problem. A detailed address on registering multiple laser scanners can be found in [14].

For the sake of temporal integration, a trigger is shot from the server computer to all client computers, at which origins of the relative time axis of client computers are defined. The data measured at the same time level from different laser scanners are transformed to the global coordinate system to make up an integrated frame.

\section{TRACKING AlgORITHM}

A tracking algorithm is developed assuming that the moving objects are the feet of normal pedestrians only. In this section, flow of the tracking process is first introduced and aimed at a global view of the algorithm. A tracking algorithm utilizing Kalman filter is then focused on, where a pedestrian's walking model based on the typical appearance of moving feet is defined.

\section{A. Flow of the Tracking Process}

A tracking algorithm is designed as shown in Fig. 4. In each iteration, server computer gather the data of moving feet in the latest range frames from all client computers and integrates them into a global coordinate system. Since there might be many points shooting upon the same foot, a process is first conducted clustering the moving points of the integrated range frame that has a radius less than a normal foot (e.g., $15 \mathrm{~cm}$ ), the center points of which are treated as foot candidates. Trajectory tracking is conducted by first extending the trajectories that have been extracted in previous frames, then looking for the seeds of new trajectories from the foot candidates that are not associated to any existing trajectories.

A tracing algorithm utilizing Kalman filter is developed extending the existing trajectories to current range frame. It will be addressed in detail in the later sections. Seeds of new trajectories are extracted in two steps. The foot candidates, who are not associated to any trajectories, are first paired up into step candidates (a possible pedestrian) if the Euclidean distance between them is less than a normal step size (e.g., $50 \mathrm{~cm}$ ). A foot candidate may belong to a number of step candidates, if there are multiple options. A seed trajectory is then extracted along a certain number $(N>3)$ of previous frames, which satisfies the following two conditions. First, two step candidates in successive frames overlap at the position of at least one foot candidate. Second, the motion vector decided by the other pair of nonoverlapping foot candidates changes smoothly along the frame sequence.

\section{B. Pedestrian's Walking Model}

When a normal pedestrian steps forward, one of the typical appearances is, at any moment, one foot swings by pivoting on 


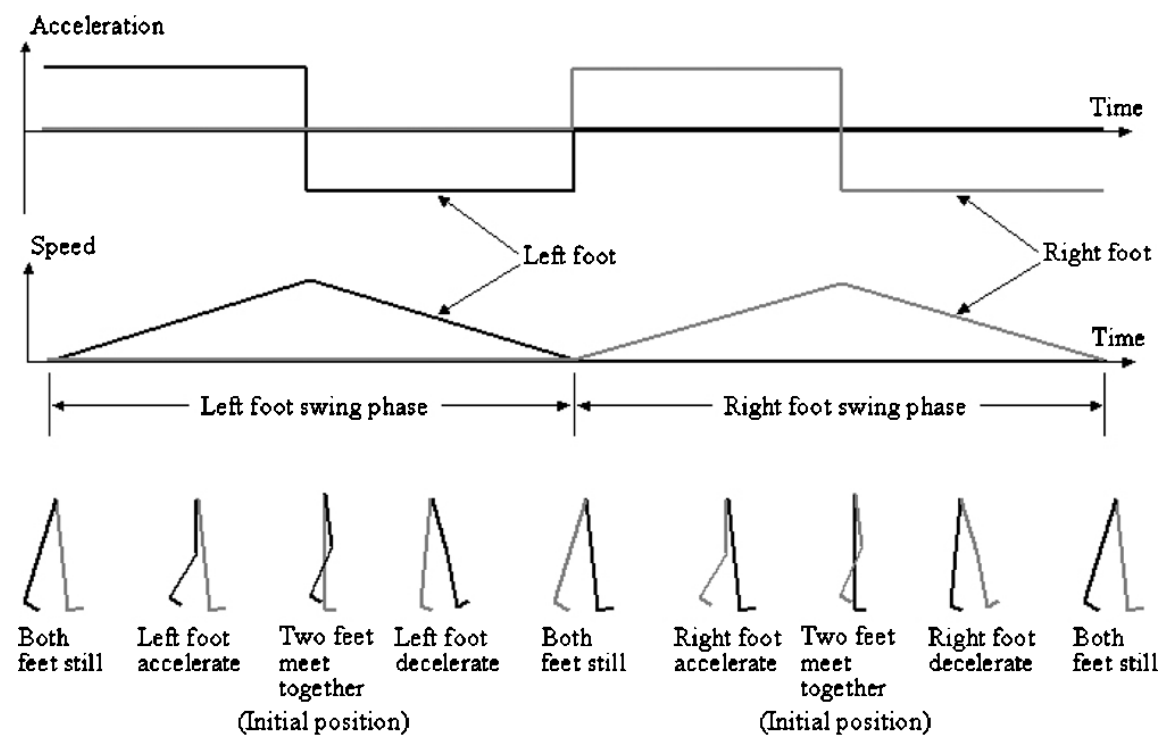

Fig. 5. A simplified pedestrian's walking model.

the other one. Two feet interchange their duty by landing and moving shifts at a rhythmic pattern.

According to the ballistic walking model proposed by Mochon and McMahon in 1980, muscles act only to establish an initial position and velocity of the feet at the beginning half of the swing phase, then remain inactive throughout the other half of the swing phase. Here, initial position refers to where swing foot and stance foot meets together. Let $v_{L}$ and $v_{R}$ be the speed, $a_{L}$ and $a_{R}$ be the accelection, $p_{L}$ and $p_{R}$ are the positions of left and right foot, respectively, where speed, acceleration, and position are restricted to a horizontal plane, and relative to the 2-D global coordinate system that has been addressed in previous sections. In the case $\left|v_{L}\right|>\left|v_{R}\right|$, where $\cdot$ is the norm of the vector, left foot swings forward by pivoting the on right foot. At the beginning half of the swing phase, the left foot shifts from rear to initial position, and swings from standing still at an accelerated speed. Here, the acceleration $\left|a_{L}\right|$ is a function of muscles strength. We define $\left|a_{L}\right|=f_{L}$ (muscle_strength) $\times \dot{v}$, where $\dot{v}$ is the normalized directional vector. During the other half of the swing phase, the left foot shifts from initial to front position, and swings with a decelerated speed from a certain speed to standing still. Here, the acceleration $\left|a_{L}\right|$ is a minus value which comes from the forces other than that of the left foot muscles. We define $\left|a_{L}\right|=-f_{L}$ (other_forces) $\times \dot{v}$. During the whole swing phase, the right foot keeps almost still, so it has $\left|v_{R}\right| \approx 0$ and $\left|a_{R}\right| \approx 0$. In the same way, we can deduce the speed and acceleration parameters when the right foot swings forward by pivoting on the left foot, where acceleration $\left|a_{R}\right|=f_{R}$ (muscle_strength) $\times \dot{v}$ and $\left|a_{R}\right|=-f_{R}$ (other_forces) $\times \dot{v}$ at the beginning and end half of swing phase, respectively, $\left|v_{L}\right| \approx 0$ and $\left|a_{L}\right| \approx 0$ during the whole swing phase. In this research, we simplify a pedestrian's walking model by assuming that the acceleration and deceleration on both feet from either muscle strength or other forces $\left(\left|a_{L \mid R}\right|\right)$ are equal and constant during each swing phase, and they have only smooth changes as the pedestrian steps forward. Fig. 5 shows an example of the simplified pedestrian's walking model.

\section{Definition of State Model}

As was described in the previous section, a pedestrian's walking model consists of three kinds of state parameters: 1) position; 2) speed; and 3) acceleration. Position and speed vectors of each pedestrian change continuously, while acceleration parameters change by swing phase in a discontinuous way. A discrete Kalman filter is designed in this research by dividing the state parameters into two vectors as follows:

$$
s_{k, n}=\Phi s_{k-1, n}+\Psi u_{k, n}+\omega
$$

where, $s_{k, n}$ consists of the positions and speed vectors of both feet of pedestrian $n$ at range frame $k$, while $u_{k, n}$ consists of the acceleration parameters. $\omega$ is the state-estimation error

$$
s_{k, n}=\left(\begin{array}{c}
p_{L \_x, k, n} \\
p_{L \_y, k, n} \\
v_{L_{-} x, k, n} \\
v_{L_{-}, k, n} \\
p_{R \_x, k, n} \\
p_{R \_y, k, n} \\
v_{R \_x, k, n} \\
v_{R \_y, k, n}
\end{array}\right) u_{k, n}=\left(\begin{array}{c}
a_{L \_x, k, n} \\
a_{L_{-} y, k, n} \\
a_{R \_x, k, n} \\
a_{R \_y, k, n}
\end{array}\right) .
$$

The transition matrix $\Phi$ relates positions and speed vectors at a previous time step to those of current one, while $\Psi$ relates acceleration values to the change in positions and speed vectors. They are defined as follows, where $\Delta t$ is the time interval between range frames. In this research, $\Delta t \approx 0.1 \mathrm{~s}$

$$
\Phi=\left(\begin{array}{cccccccc}
1 & 0 & \Delta t & 0 & 0 & 0 & 0 & 0 \\
0 & 1 & 0 & \Delta t & 0 & 0 & 0 & 0 \\
0 & 0 & 1 & 0 & 0 & 0 & 0 & 0 \\
0 & 0 & 0 & 1 & 0 & 0 & 0 & 0 \\
0 & 0 & 0 & 0 & 1 & 0 & \Delta t & 0 \\
0 & 0 & 0 & 0 & 0 & 1 & 0 & \Delta t \\
0 & 0 & 0 & 0 & 0 & 0 & 1 & 0 \\
0 & 0 & 0 & 0 & 0 & 0 & 0 & 1
\end{array}\right)
$$




$$
\Psi=\left(\begin{array}{cccc}
0.5 \Delta t^{2} & 0 & 0 & 0 \\
0 & 0.5 \Delta t^{2} & 0 & 0 \\
\Delta t & 0 & 0 & 0 \\
0 & \Delta t & 0 & 0 \\
0 & 0 & 0.5 \Delta t^{2} & 0 \\
0 & 0 & 0 & 0.5 \Delta t^{2} \\
0 & 0 & \Delta t & 0 \\
0 & 0 & 0 & \Delta t
\end{array}\right)
$$

In addition, the state vector $u_{k, n}$ is predicted by identifying the swing phase as follows, where $\dot{v}$ is the normalized directional vector.

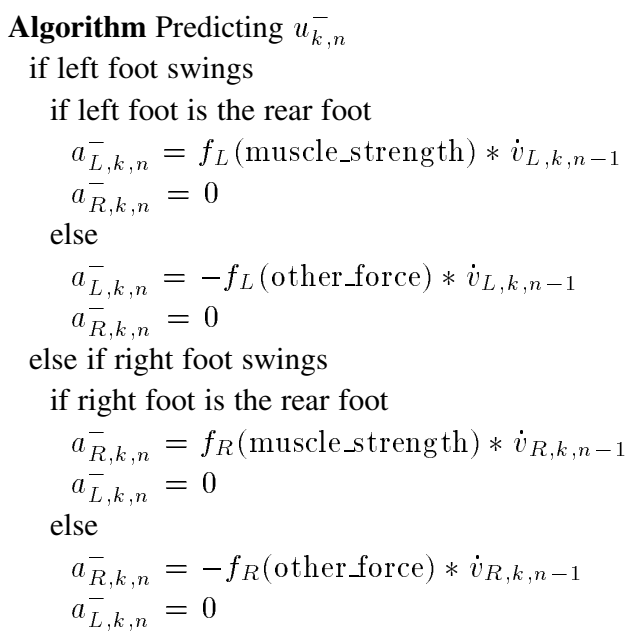

As has been addressed previously, in this research we assume the acceleration on both feet from either muscle strength or other forces are equal and constant during each swing phase, and it changes smoothly as the pedestrian steps forward. The acceleration function is updated whenever a cycle of left foot and right foot swing phase is finished, and it is conducted by taking the mean of the change of a number of latest swing phases. Suppose $N$ latest swing phases have been counted from time steps $j$ to $k$, the acceleration function is calculated as follows, where $S_{L \mid R}$ is the average ofthe left or right foot's step size.

$$
\begin{aligned}
S_{L \mid R} & =\frac{\sum\left|p_{L \mid R, k, n}-p_{L \mid R, k-1, n}\right|}{N} \\
f_{L \mid R} & =\frac{S_{L \mid R}}{(k-j+1)^{2} * \Delta t^{2}} .
\end{aligned}
$$

The discrete Kalman filter updates the state vector of $s_{k, n}$ based on the measurements as follows:

$$
m_{k, n}=\mathbf{H} s_{k, n}+\epsilon
$$

where $m_{k, n}$ denotes the measurements of pedestrian $n$ at time step $k, H$ relate the state vector $s_{k, n}$ to measurements $m_{k, n}$, and $\epsilon$ represents the measurement error

$$
m_{k, n}=\left(\begin{array}{l}
p_{L \_x, k, n} \\
p_{L \_y, k, n} \\
p_{R \_x, k, n} \\
p_{R \_y, k, n}
\end{array}\right)
$$

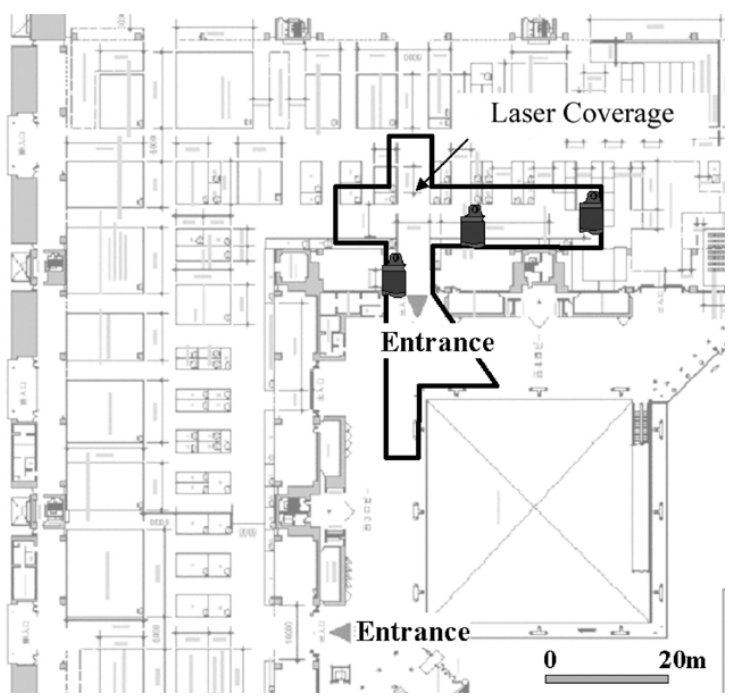

Fig. 6. Layout of laser scanners and their coverage at an exhibition hall.

$$
\mathbf{H}=\left(\begin{array}{llllllll}
1 & 0 & 0 & 0 & 0 & 0 & 0 & 0 \\
0 & 1 & 0 & 0 & 0 & 0 & 0 & 0 \\
0 & 0 & 0 & 0 & 1 & 0 & 0 & 0 \\
0 & 0 & 0 & 0 & 0 & 1 & 0 & 0
\end{array}\right)
$$

\section{Tracing Process Using Kalman Filter}

In tracking each trajectory, the state vector $u_{k, n}$ is first predicted by identifying the swing phase, $s_{k, n}$ and $m_{k, n}$ are then predicted according to (1) and (7), respectively. A searching area is defined on the predicted $m_{k, n}$. If foot candidates of the current frame are found inside the search area, the nearest ones are exploited to compose the updated $m_{k, n}$. Otherwise, missing counter starts. If the missing counter is larger than a given threshold, e.g., 20 frames $(\approx 2 \mathrm{~s})$, then the tracing of the trajectory stops. Otherwise, the predicted $m_{k, n}$ is exploited as the updated one to update the state vector $s_{k, n}$ and Kalman gain. The process continues until all the trajectories are traced.

\section{EVALUATION OF THE System}

The system is evaluated in two levels, real experiments and computer simulations. Through real experiments, feasibility of the system in tracking pedestrians in an open wide environment is examined. On the other hand, since there is still no method to find the true trajectories of a large crowd and in a wide area, evaluation through computer simulation aims at quantitatively examining the performance of the system, e.g., how many LD-As are needed to cover a certain area, what percentage of the pedestrians are extracted with respective to different crowd density, how accurate are they, and so on.

\section{A. Evaluation Through a Real Experiment}

An experiment is conducted, where three LD-As are exploited, covering a corner of an exhibition hall, and near to one of its entrances. Fig. 6 shows the layout and laser coverage of the sensor system. Laser coverage here means the area is measured by at least one laser scanner, considering only the occlusions from the physical layout of the environment. Laser 


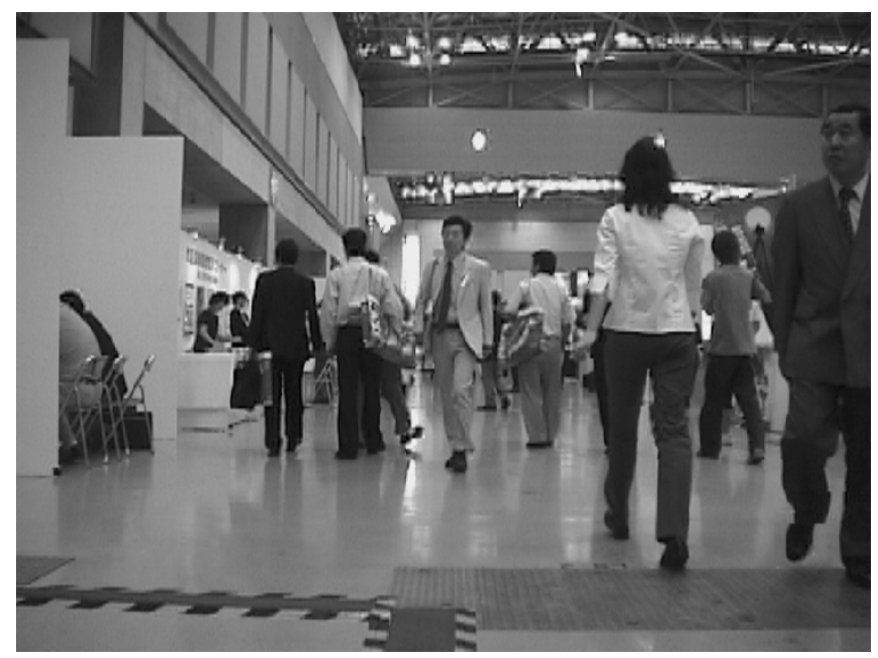

Fig. 7. Photo of experimental site captured from a viewpoint just above the laser scanner.

scanning is conducted at an elevation of about $20 \mathrm{~cm}$ above the ground. Fig. 7 is an photo of the experiment site, which is taken by putting a camera on the top of a LD-A. LD-As are located at different places, about $20 \mathrm{~m}$ far from each other, covering an area of about $60 \times 60 \mathrm{~m}^{2}$. Four computers are exploited in the experiment, where three with Pentium III CPU of $600 \mathrm{MHz}$ control LD-As, and one with Pentium IV CPU of $1.0 \mathrm{GHz}$ works as the server computer. Four computers are connected using a 10/100 Base LAN cable, composing a local network. Background images are generated previously before the opening of exhibition by each client computer, and sent to the server computer for two purposes. First, they are registered to find transformation matrices from each sensor's local coordinate system to a predefined global one. Second, they are shown as well as the data of moving feet that are recorded in each scan for a real-time monitoring.

The server computer exploited in this research is able to do a real-time extraction up to 30 trajectories simultaneously due to the limitation of processing power. Whereas, in the environment we test, there are much more visitors. In this experiment, visitor's trajectories are tracked through a postprocessing. Fig. 8 is a screen copy of reproduced trajectories, where opaque circles with a white background denote the location of LD-As; grey points represent background image; white points represent moving feet; lines are the trajectories during the latest 50 range frames; transparent circles mark the grouped foot candidates. There is some mistracking in the experiment. In the case that visitors carry large bags, the data of which are also measured by LD-As and recognized as additional feet. In the case that visitors cross and their feet are too close together at intersection point, the data of which are mixed and one foot is lost in extraction. The tracking algorithm will be improved in a future study to figure out such problems.

The experiment lasted a whole day. Changes in visitor's flow are examined. In the following, we show an example of analyzing the change in visitor's number along with time. Three pieces of measurements are exploited, which are taken at 10:00 pm, 1:00 am, and 3:00 am, respectively, and each lasts for 10 minutes. Visitor's trajectories are divided into two groups. If there is only a slight change in position during the period of measurement, e.g., less than $1 \mathrm{~m}$, the trajectory is treated as a still one; otherwise, it is treated as a walking one. Trajectories are counted, and their average numbers are shown in Fig. 9. Tendency in the change of visitor's number can be grasped.

\section{B. Evaluation Through Computer Simulation}

A computer simulation is conducted to quantitatively evaluate system performance for tracking pedestrians in an open wide environment. Flow for generating synthesized laser scans through computer simulation is shown in Fig. 10. They are explained in details as follows.

A digital map of the exhibition hall is loaded defining the physical layout, such as building blocks and poles, of the surroundings, using closed polygons. Since the showrooms in the exhibition hall are open ones, and most of the visitors inside the showroom stand still for a long time watching and talking, in this simulation, we close the showrooms, and simulate only the pedestrians that walk on the passageway. Three laser scanners with the same specification of LD-As are set and a rectangular walking area of $73 \times 53 \mathrm{~m}$ for simulated pedestrians to move around are defined according to the real experiment. The number of pedestrians in the walking area is set previously and kept constant during the whole simulation. It means that whenever a simulated pedestrian walks to the outside of the walking area or to the inside of a building block (e.g., showroom), it "dies out" from the simulation, meanwhile a new pedestrian is created. Fig. 11 shows a screen copy of a simulation, where the number of pedestrians is set to 50. As the resolution of laser scanning becomes sparse to measure a normal foot, we reduce the range of laser scanner 70 to $30 \mathrm{~m}$ to save computation cost. The following evaluations consider only the trajectories inside laser coverage. In the case shown in Fig. 11, except the building blocks, $57 \%$ of the walking area is covered by laser scans. Among the total of 50 pedestrians, about 30 pedestrians walk inside the area of laser coverage. Occlusion caused by the 30 pedestrians (the shadowed area behind each pedestrian) is about $3.9 \%$ of the total laser coverage, blocking the measurement of other pedestridans.

Pedestrian walking is simulated exactly according to the simplified pedestrian's walking model that is defined in previous section. Besides the state vectors recording position, speed and acceleration, pedestrians are characterized using additional three parameters, foot radius $(\approx 0.15 \mathrm{~m})$, step size $(\approx 0.7 \mathrm{~m})$, and walking speed $(\approx 5.0 \mathrm{~km} / \mathrm{h})$. Parameters of each pedestrian are initialized at the beginning of the simulation to the posture that both feet keep still, the left foot is the rear one and has an acceleration toward to the right foot. Position as well as walking direction (normal vector) of the left foot is initialized in a random way. The norm of acceleration is calculated according to pedestrian's step size and walking speed as defined in (6).

A timer is set according to the scanning rate of laser scanners. Parameters of simulated pedestrians are updated at each time step (every $0.1 \mathrm{~s}$ in this smulation). In order to give variation to walking trajectories and walking patterns, an adjustment to step size as well as walking speed is assigned at the start of each swing phase, and a change in walking direction is given when 


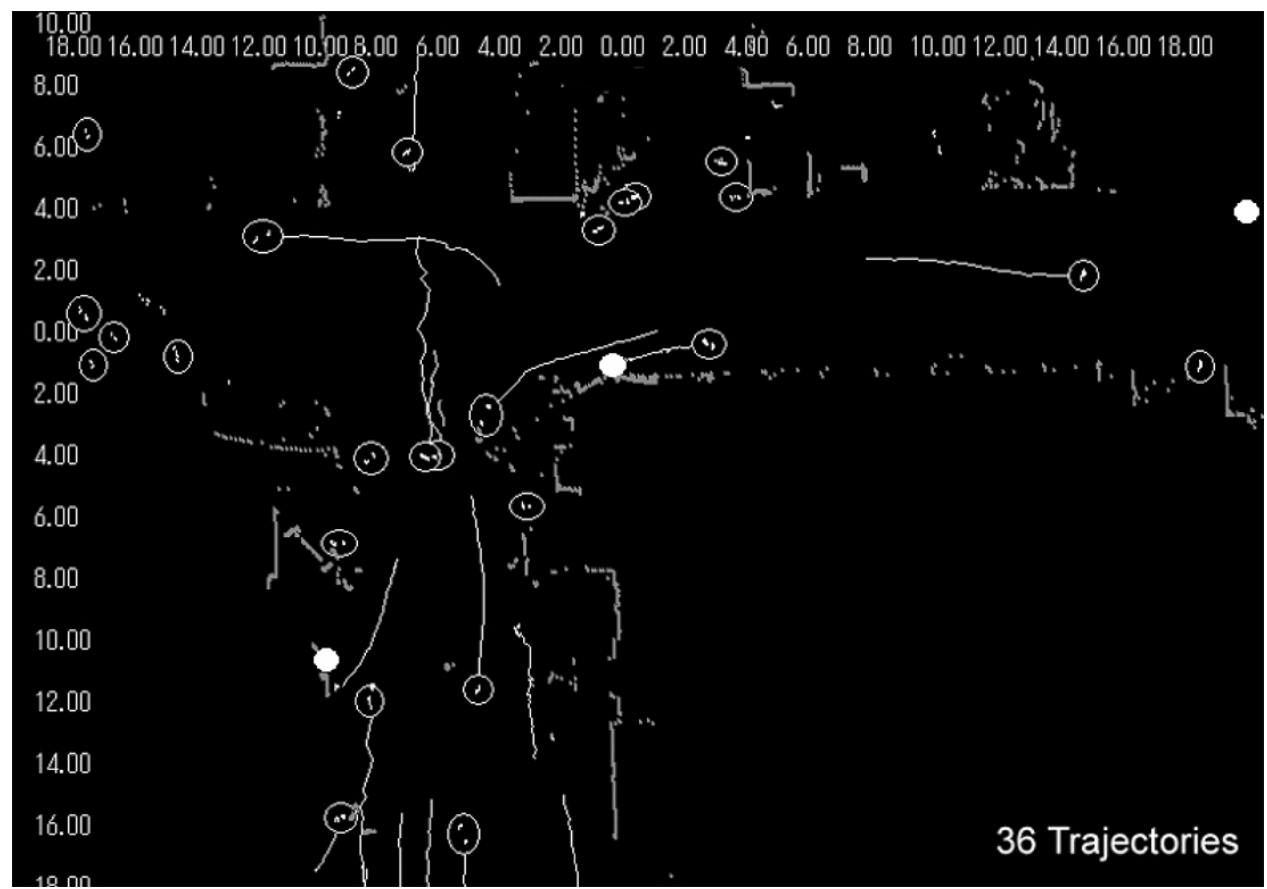

Fig. 8. Screen copy of reproduced trajectories.

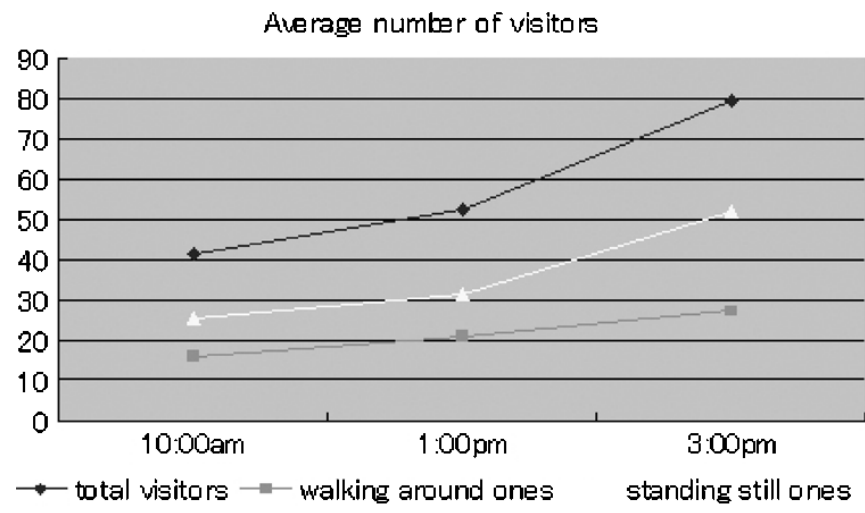

Fig. 9. Change of visitor's number along with time.

both feet meet at the same position. Also, laser scans are simulated for each laser scanner at each time step. Range values are assigned by the distances from the laser scanner to the nearest object along the direction of the laser beams. They are flagged to "moving object," "still object," and "infinity," according to the objects that laser beam meet. Here, the boundary of a foot is considered as a circle with a predefined foot radius. The data of moving objects are transformed to a global coordinate system composing an integrated frame.

Trajectories are tracked and pedestrians are counted from the synthesized laser scans using the algorithm addressed in previous sections. They are compared with the true trajectories of simulated pedestrians. Fig. 12 shows an evaluation of a tracking result, where the simulation lasts for $45 \mathrm{~s}$ and 103 true trajectories are generated during the simulation. In Fig. 12, black lines are the extracted trajectories, while white lines underneath are the true ones. It can be found that although some of the trajectories are broken into pieces, all of the total 103 trajectories are extracted, even partially. By matching trajectory

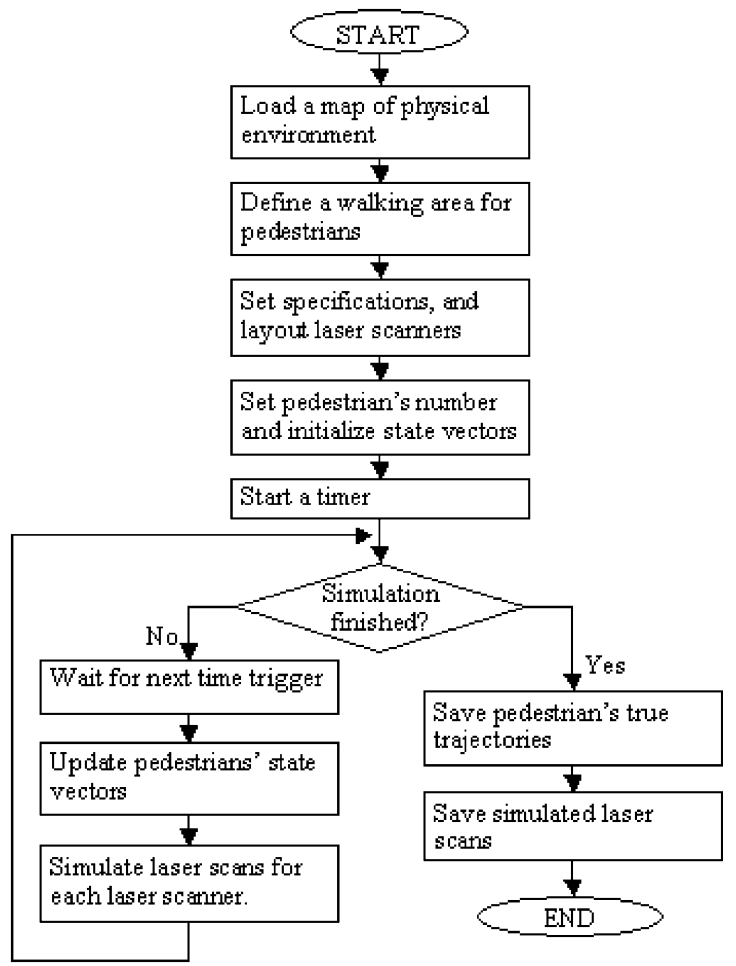

Fig. 10. Flow for generating synthesized laser scans through computer simulation.

pixels (position of the trajectory at each time step), $84.8 \%$ of the pixels of true trajectories found their matched pairs in extracted ones. The residual between matched trajectory pixels are $8.7 \mathrm{~cm}$. In addition, pedestrians are counted in Fig. 13. When 29.4 pedestrians walk under laser coverage, an average of 2.1 pedestrians are blocked by others. Using the tracking algorithm, 24.9 pedestrians are counted. Several simulation 


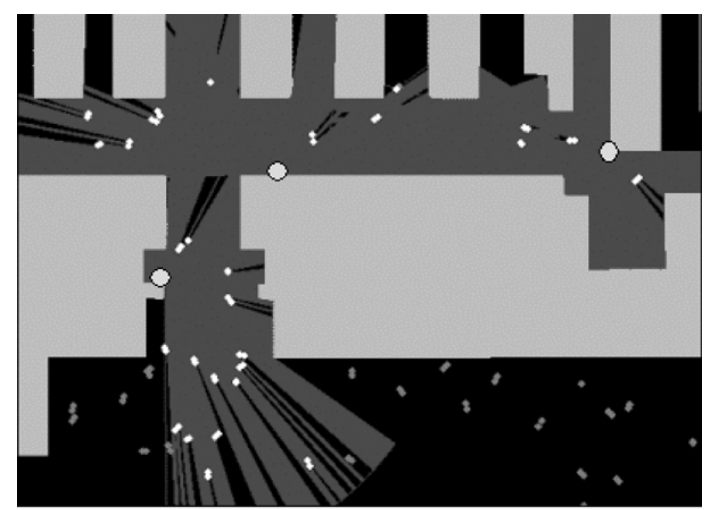

Laser scanner

B Both feet are visible to laser scanners

$\square$ One foot is visible to laser scanners

No foot is visible to laser scanners

Block of the building wall

Inside Laser coverage

Out of laser coverage

Fig. 11. Computer simulation of pedestrian's walking and laser scanning in a predefined enviroment.

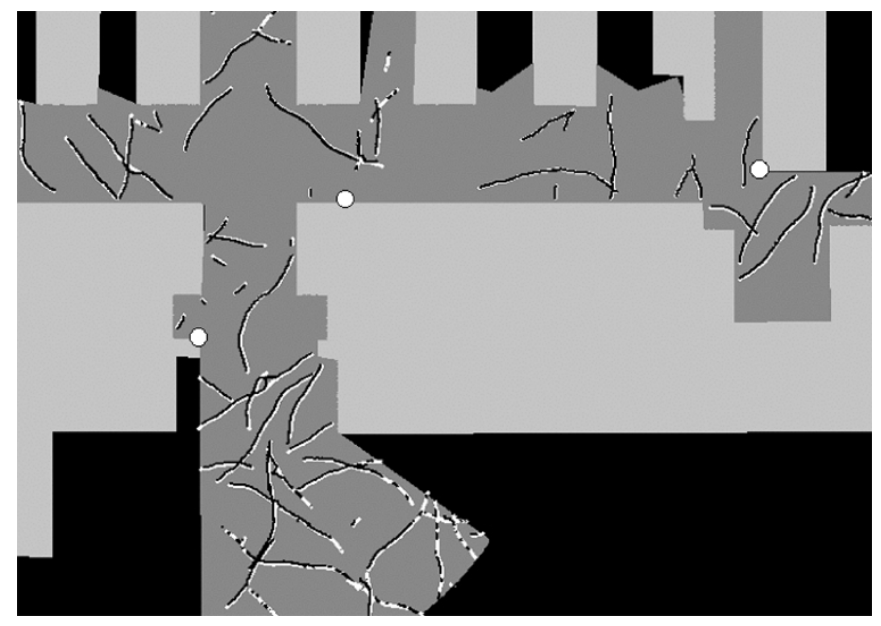

Fig. 12. Comparison of extracted trajectories with true values.

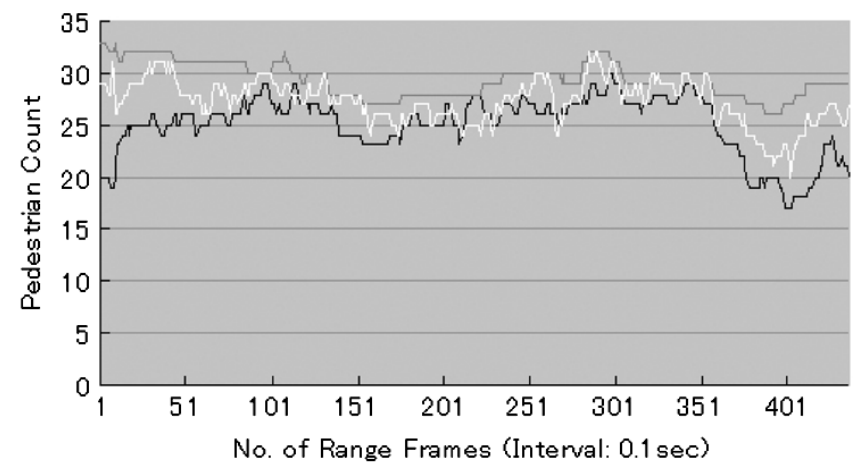

- counted by the system — total pedestrians

observable to laser

Fig. 13. Evaluation of a pedestrian's counting results.

experiments are conducted by changing the number of pedestrians in the walking area, the results of tracking trajectories and counting pedestrians with respect to different crowd densities are compared in Figs. 14 and 15.

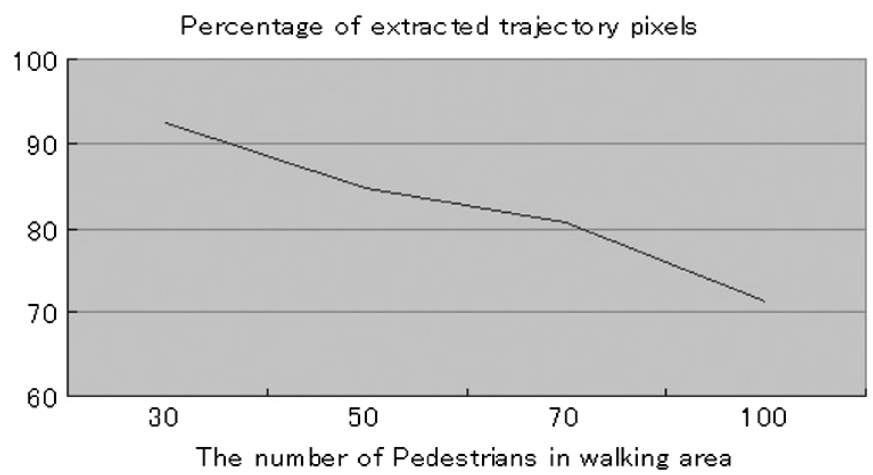

Fig. 14. Comparison of a trajectory's tracking results with respect to crowd density.

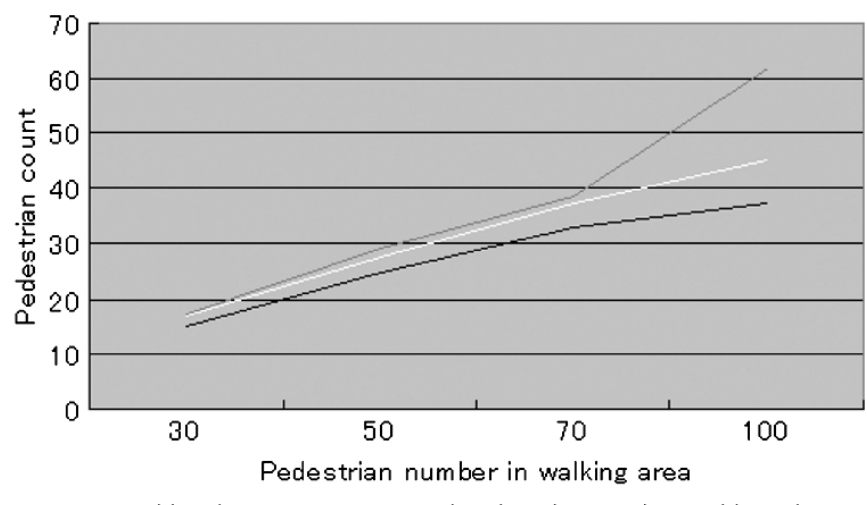

Fig. 15. Comparison of a pedestrian's counting results.

\section{CONCLUSION}

In this paper, a novel system is proposed of tracking pedestrians in a wide and open area, such as a shopping mall and an exhibition hall, by scanning the feet of pedestrians using a number of single-row laser-range scanners. The system is evaluated in two levels, real experiment and computer simulation. In real experiment, three laser scanners are set on an exhibition hall, monitoring visitors' flow during a whole exhibition day. About $60 \times 60 \mathrm{~m}^{2}$ are covered, and more than 100 visitors are tracked simultaneously during a peak hour. Changes in visitors' flow are also examined using the extracted trajectories. In addition, a computer simulation is conducted to quantitatively evaluate the performance of the system. Although the tracking algorithm is still not robust and accurate enough to follow each individual and track the complete trajectories of a large crowd, the efficiency of the system in examining pedestrian's flow and finding their tendency in a wide and open area is proved. Comparing the tracking using normal video cameras, it can be concluded that the method using single-row laser-range scanners has the following advantages. First, it is a kind of direct measurement. Extraction of moving objects in real-world coordinate system is not such a time-consuming work as that of using normal video camera. Second, as laser scan can be easily converted into a rectangular coordinate system of real dimension on a horizontal plane, it is relatively easily to calibrate multiple laser scanners and integrate distributed data to cover a relatively large area, Third, tracking of large crowd can be achieved in a real-time way in the near future due to the low computation cost. 
Finally, although range measurement has poor interpretability compared to video image, to some extent, it avoids the "privacy problem," which is a rather sensitive topic in supermarket, exhibition hall, etc.

In future study, extending pedestrian's walking model to a more realistic one, e.g., applying the ballistic walking models, which have been widely studied in the field of biomechanics (e.g., [10]), and refining the tracking method as well as the simulation system to achive higher accuracy while keep low computation cost will be addressed. In addition, a tracking algorithm will be developed for monitoring not only pedestrians, but also shopping carts, baby cars, bicycles, motor cars, and so on.

\section{REFERENCES}

[1] A. Fod, A. Howard, and M. J. MataricL, "Laser-based people tracking," in Proc. IEEE Int. Conf. Robot. Autom., Washington, DC, 2002, pp. 3024-3029.

[2] D. Gavrila, "The visual analysis of human movement: A survey," Comput. Vision Image Understand., vol. 73, no. 1, pp. 82-98, 1999.

[3] D. S. Jang, G. Y. Kim, and H. I. Choi, "Model-based tracking of moving object," Patt. Recogn., vol. 30, no. 6, pp. 999-1008, 1997.

[4] S. Mochon and T. A. McMahon, "Ballistic walking," J. Biomech., vol. 13, pp. 49-57, 1980.

[5] C. J. Pai, H. R. Tyan, Y. M. Liang, H.-Y. M. Liao, and S. W. Chen, "Pedestrian detection and tracking at crossroads," Patt. Recogn., vol. 37, no. 5, pp. 1025-1034, 2004.

[6] E. Prassler, J. Scholz, and A. Elfes, "Tracking people in a railway station during rush-hour," in Proc. ICVS, H. I. Christensen, Ed., 1999, pp. $162-179$.

[7] C. S. Regazzoni and A. Tesei, "Distributed data fusion for real-time crowding estimation," Signal Process., vol. 53, pp. 47-63, 1996.

[8] C. Sacchi, G. Gera, L. Marcenaro, and C. S. Regazzoni , "Advanced image-processing tools for counting people in tourist site-monitoring applications," Signal Process., vol. 81, pp. 1017-1040, 2001.

[9] T. J. Schofield, A. J. Stonham, and P. A. Mehta, "Automated people counting to aid lift control," Autom. Construction, vol. 6, pp. 437-445, 1997.

[10] R. W. Selles, J. B. J. Bussman, R. C. Wagenaar, and H. J. Stam, "Comparing predictive validity of four ballistic swing phase models of human walking," J. Biomech., vol. 34, pp. 1171-1177, 2001.
[11] D. Streller, K. Furstenberg, and K. C. J. Dietmayer, "Vehicle and object models for robust tracking in traffic scenes using laser-range images," in Proc. IEEE 5th Int. Conf. Intell. Transport. Syst., Sept. 2002, pp. 118-123.

[12] K. Uchida, J. Miura, and Y. Shirai, "Tracking multiple pedestrians in crowd," in Proc. IAPR Workshop Mach. Vision Applicat., Nov. 2000, pp. 533-536.

[13] G. Welch and G. Bishop, "An Introduction to the Kalman Filter," Univ. North Carolina, Chapel Hill, Tech. Rep. TR95-041, Feb. 2001.

[14] H. Zhao and R. Shibasaki, "A robust method for registering groundbased laser range images of urban outdoor environment," Photogram. Eng. Remote Sensing, vol. 67, no. 10, pp. 1143-1153, 2001.

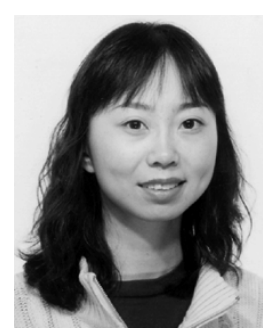

Huijing Zhao was born in Beijing, China. She received the B.S. degree in computer science from Peking University, Beijing, China, in 1991 and the M.E. and Ph.D. degrees in civil engineering from the University of Tokyo, Japan, in 1999 and 1996, respectively.

From 1991 to 1994, she was with Peking University, working on a project developing a GIS platform. After several years of conducting post-doctoral research, she is now a Visiting Associate Professor in the Center for Spatial Information Science, University of Tokyo, Tokyo, Japan. Her research interest covers image sensing, computer vision, and spatial data handling for GIS applications.

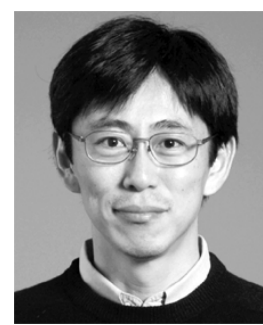

Ryosuke Shibasaki (M'98) was born in Fukuoka, Japan. He received the B.S., M.S., and Doctoral degrees in civil engineering from the University of Tokyo, Tokyo, Japan, in 1980, 1982, and 1987, respectively.

From 1982 to 1988 , he was with the Public Works Research Institute, Ministry of Construction. From 1988 to 1991, he was an Associate Professor in the Civil Engineering Department, University of Tokyo. In 1991, he joined the Institute of Industrial Science, University of Tokyo. In 1998, he was promoted to a Professor in the Center for Spatial Information Science, University of Tokyo. His research interest covers three-dimensional data acquisition for GIS, conceptual modeling for spatial objects, and agent-based microsimulation in GIS environment. 